\title{
A Brief Parental Education for Shaping Sleep Habits in 4-Month-Old Infants
}

\author{
Yoshiko Adachi, MD, PhD; Chifumi Sato, MD, PhD; Noriko Nishino, MD; Fumitake Ohryoji, MD; \\ Junko Hayama, MA; and Toshiko Yamagami, MD, PhD
}

\begin{abstract}
Objective: To examine the effectiveness of a simple behavioral intervention with an educational booklet on the modification of parenting behaviors and the prevention of sleep disturbance in 4-month-old infants.
\end{abstract}

Design: A prospective cohort design with cluster sampling controls in a primary care setting.

Participants: A total of 136 mothers with 4-month-old infants who visited a local health check-up clinic and responded to the 3-month follow-up survey.

Methods: At the health check-up, an intervention group was provided with 10 minutes of group guidance and a simple educational booklet designed to encourage parents to promote favorable sleep patterns in their infants.A control group was provided with standard education alone. The two groups were questioned 3 months later through a survey sent by mail. Main measurements were parenting behaviors of parents and night waking of infants.

Results: Two undesirable maternal behaviors that reinforce night waking in infants exhibited a significantly greater improvement in the intervention group than in the control group. "Feed or check diaper promptly" behavior and "hold and soothe immediately" behavior after "night waking" in the infant were significantly decreased in the intervention group.

Conclusions: Our findings suggest that increased night waking in infants was prevented in the intervention group. Our results indicate that the proportion of infant night waking was significantly higher in the control group, and there was also a tendency toward an increase in the proportion of infants who woke frequently and cried. These infant behaviors were not changed in the intervention group at the 3-month follow-up stage.

Key words: Community health services, Early intervention, Infant, Parenting education, Sleep

\begin{abstract}
A problems and night wakings. ${ }^{1,2}$ Enduring infant and toddler sleep disturbance (ITSD), which includes "difficulty settling" or "night waking," may negatively affect the health of children and parents. ${ }^{3-6}$ Compared to research on non-drug intervention methods for treating sleep problems in adults, ${ }^{7,8}$ research on intervention strategies for treating infant sleep problems has been relatively sparse. Recently, behavioral interventions have increasingly been recognized as a treatment of choice for ITSD. ${ }^{1,2,9}$ Empirically supported behavioral interventions ${ }^{10}$ include extinction ${ }^{11}$ and its variants, ${ }^{12,13}$ positive bedtime routine, ${ }^{12}$ scheduled awakenings, ${ }^{14}$ and disassociating feeding from sleep-wake transitions. These methods were developed
\end{abstract}

on the basis of the hypothesis that ITSD is maintained or reinforced by prompt parental behaviors in response to infant night wakings, such as feeding, holding, and soothing.

Mindell ${ }^{1}$ reviewed 41 studies and reached the conclusion that extinction and parental education should be considered as well-established methods for the treatment of sleep problems. Ramchandani and colleagues ${ }^{9}$ have described how appropriate behavioral interventions can offer long-lasting benefits for children and families. On the basis of a review of 52 studies, the American Academy of Sleep Medicine ${ }^{2}$ has concluded that behavioral therapy produces reliable and long-lasting effects. This provides strong support for unmodified extinction
Corresponding Author: Yoshiko Adachi, MD, PhD, 3-29-II Ishizaka, Dazaifu-city, Fukuoka 818-0I I 8, Japan, Tel: +8I-92-919-57|7, Fax: +81-92-928-9522, Email:a_ibh@ybb.ne.jp
Received: August 3, 2008

Ist Revision: October 30, 2008

2nd Revision: February 2, 2009

Accepted: February II, 2009

doi: $10.3121 / \mathrm{cmr} .2009 .814$

\section{Disclosure:}

This research was supported by the Mental Health Okamoto Memorial

Foundation. 


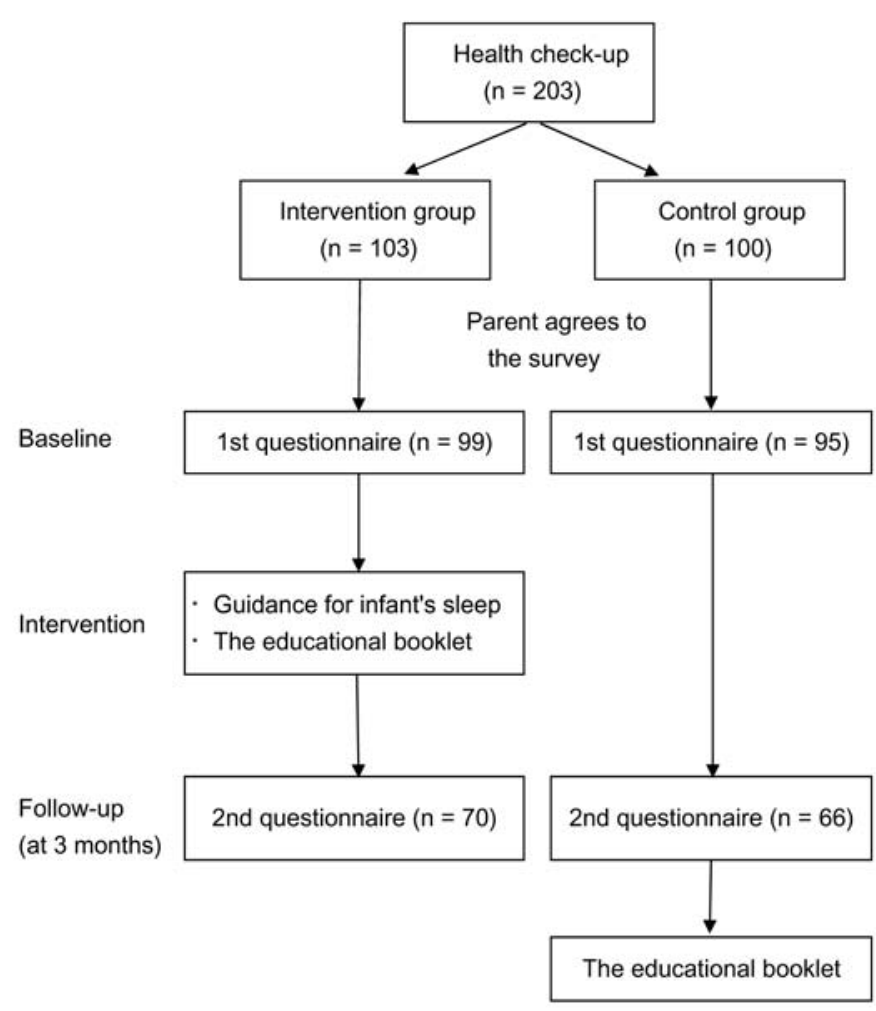

Figure 1. Flow diagram of study design.

approaches and prevention through parental education. Although it has been suggested that unmodified extinction is the most effective method for reducing ITSD, there are some practical difficulties associated with this approach. Without attending to their infants when they cry during the night, many parents experience feelings of guilt. These parents are unable to wait until their infants go back to sleep before attending to them again. Considering the difficulties in correcting parental behaviors and reversing the associated learning mechanism in infants that exacerbates ITSD, parental education prior to the development of frequent night waking is advocated as the most acceptable and effective solution. Straightforward educational approaches aimed at preventing ITSD have shown promising results. Several reports have provided strong evidence that education using behavioral booklets is an effective strategy for reducing ITSD incidence. ${ }^{3,13,15,16}$

There have been several studies on the effects of education as an early intervention strategy during the neonata ${ }^{15}$ and pregnancy ${ }^{17,18}$ stages of parenthood. In many of these studies, outcome measures related to infant sleep and maternal distress have been evaluated. However, there is little data concerning parental behavioral changes after intervention. Because the key focus of education strategies aimed at reducing ITSD is on practical parental behaviors, and considering that parental behavioral patterns play a key role in shaping infant sleep behavior, an in-depth understanding of parental behavior is highly desirable. Establishing effective educational strategies that promote healthy sleep patterns in infants will be beneficial to the mental health of infants and parents. To accomplish this, implementation of suitable educational programs in primary care settings is a necessary requirement.

In Japan, the relationship between parental behavior and infant sleep problems is not clearly understood. Indeed, research has been lacking into behavioral interventions that address sleep problems in infants. Furthermore, infants sleeping with their parents is common in Japan and is traditionally accepted. ${ }^{19,20}$ In the current infant health check-up system, the Mother and Child Health Law makes no provision for information or education for parents on infant sleep patterns.

We previously reported that 4-month-old infants go to bed late (mean bedtime: 10:28 PM) and demonstrated a high night waking rate of $70 \%$ in a questionnaire-based investigation in Chuo-ku, Fukuoka City, Japan. ${ }^{21}$ In the same subjects, we showed clear relationships between undesirable parenting and infant sleep problems, and found by using path analysis that sleep difficulties in infants contribute to sleep disturbances and associated health problems in the mother. Additionally, $30 \%$ of the mothers questioned were suspected of having some type of sleep disorder, and more than half the mothers had undesirable parenting habits considered detrimental toward healthy infant sleep. ${ }^{22}$ In view of these concerns, we tested an intervention strategy that involved providing mothers with a simple educational booklet about infant sleep and parental behavior during their 4-month-old infant health check-up session.

In this study, we sought to accomplish two major aims. Our first aim was to determine if parental behaviors in mothers were modified by simple informational content on behavior provided in the booklet. Second, we sought to evaluate the efficacy of this intervention strategy in preventing sleep problems in 4-month-old infants. To examine educational effects, the intervention group was compared with a control group over a 3-month period.

\section{Methods}

The study subjects consisted of mothers and infants who visited a health check-up session for 4-month-old children at the Fukuoka Chuo Health Center in January and February 2005. This check-up is performed for all residents as a health service that is provided in accordance with the Mother and Child Health Law. The same subjects that participated in previous cross-sectional research studies ${ }^{21,22}$ were evaluated in this study. This study was conducted in collaboration with the Center for Public Health and Welfare, Chuo-ku, Fukuoka City.

\section{Study Protocol}

This study used a prospective cohort design with cluster sampling controls (figure 1). The 3-month research protocol was approved by the Ethics Committee of the Association for Preventive Medicine of Japan. Of 203 participants invited to 
Table 1. Baseline characteristics of the study participants.

\begin{tabular}{|c|c|c|c|}
\hline & $\begin{array}{l}\text { Intervention group } \\
\qquad(\mathrm{n}=99)\end{array}$ & $\begin{array}{l}\text { Control group } \\
(n=95)\end{array}$ & $P$-value* \\
\hline \multicolumn{4}{|l|}{ Infants } \\
\hline Gender, \% male & 50.50 & 54.70 & NS \\
\hline Mean gestation weeks & 39.23 & 38.99 & NS \\
\hline Mean birth weight, $g$ & 3027.22 & 2950.94 & NS \\
\hline \multicolumn{4}{|l|}{ Parents } \\
\hline Maternal mean age, y & 31.72 & 31.55 & NS \\
\hline Paternal mean age, y & 34.84 & 34.27 & NS \\
\hline Maternal occupation, \% present & 24.2 & 30.3 & NS \\
\hline Married, \% present & 99.0 & 97.9 & NS \\
\hline
\end{tabular}

${ }^{*} \chi^{2}$ test was performed for comparison of proportion and student's $t$ test was performed for comparison of means.

participate, 196 (194 mothers, 1 father, and 1 grandmother) agreed to provide answers to our questionnaire. Informed consent was obtained from each study participant. The actual response rate was $96.6 \%$ for completion of the questionnaire. The subjects in the intervention group were recruited in January, while those in the control group were recruited in February. Following completion of the questionnaire, subjects were also assessed with a follow-up questionnaire 3 months later.

\section{Intervention and Control Groups}

Figure 1 shows a flow diagram of participants as they progressed through the various stages of the study. A total of 103 participants who visited the health check-up session were invited to participate in the survey as intervention group study subjects. Among them, 99 mothers agreed to participate and provided answers for the baseline stage of the questionnaire. These individuals received a booklet entitled "Baby, Sleep Well at Night" 23 following the health check-up and then participated in a brief 10-minute group presentation (delivered by the first author) that provided guidance on infant sleep. The booklet was written as part of a review report on behavioral strategies to improve infant sleep problems. ${ }^{24}$ The table of contents from the booklet are presented in appendix 1 of this article. It consists of 20 pages providing educational information on practical behaviors that have previously been outlined in publications by Kerr and Jowett, ${ }^{25} \mathrm{Schmitt}^{26}$ and Eckerberg. ${ }^{13}$ The booklet provides content regarding the theoretical background and reasons believed to be responsible for night waking. The booklet also contains information on the development of circadian rhythms in newborns as well as advice regarding optimal environmental conditions and parental behavioral patterns for encouraging healthy sleep habits of infants. In addition, information is also provided on the behavioral principle of operant conditioning. A list of recommended behavioral strategies is presented in the booklet that aims to promote extinction of night waking and improve difficulties in settling to sleep. Among these behavioral interventions, desirable parental strategies include waiting for a short time without responding when the infant cries during the night and silently checking the bed and clothes of the infant. In contrast, behaviors listed as undesirable include prompt feeding or immediate checking of the infant's diaper following night waking as well as prompt holding and soothing. In order to appropriately shape circadian rhythms and minimize the likelihood of bedtime settling problems, several behaviors were recommended: increasing daytime play activities involving abundant visual and auditory stimulation, establishing a regular waking time and bedtime, avoidance of exertive activities later in the evening, establishing a designated place for sleeping at a specific time, setting a bedtime routine, and encouraging the infant to fall sleep alone.

One hundred participants who visited the health check-up center enrolled in the control group. Ninety-five mothers agreed to participate in the survey and completed the questionnaire. These participants received no information on infant sleep and were provided with only the standard parenting educational information that is supplied to all visitors at the health checkup session. General information included advice on feeding, bathing, vaccinations. The control group participants received the educational booklet after the follow-up phase of the survey had been completed.

\section{Data Collection}

A follow-up questionnaire was sent to participants by postal mail at 3 months following completion of the baseline questionnaire. The questionnaire (appendix 2) was designed to evaluate sleep patterns and sleep problems in infants and mothers, parental behaviors that occurred in response to infant night waking, parental behaviors directed at efforts to promote desirable sleep patterns in infants, parental difficulties with and feelings about caring for the infant. Seventy mothers in the intervention group (70.7\%) and 66 mothers in the 
Table 2. Parental behavioral changes.

\begin{tabular}{|c|c|c|c|c|c|c|c|c|c|c|}
\hline & \multicolumn{5}{|c|}{ Intervention group $(\mathrm{IG})(\mathrm{n}=\mathbf{7 0})$} & \multicolumn{5}{|c|}{ Control group $(C G)(n=66)$} \\
\hline & \multicolumn{2}{|c|}{ Baseline } & \multicolumn{2}{|c|}{ Follow-up } & \multirow[b]{2}{*}{$P$-value } & \multicolumn{2}{|c|}{ Baseline } & \multicolumn{2}{|c|}{ Follow-up } & \multirow[b]{2}{*}{ P-value } \\
\hline & $\mathbf{N}$ & $\%$ & $\mathbf{N}$ & $\%$ & & $\mathbf{N}$ & $\%$ & $\mathbf{N}$ & $\%$ & \\
\hline \multicolumn{11}{|c|}{ Parental behaviors when infant's wake and cry at midnight (multiple answers, $n=131$; IG = 66; CG =65) } \\
\hline Wait for awhile without responding & 33 & $50.0^{*}$ & 42 & 63.6 & 0.108 & 20 & 30.8 & 32 & 49.2 & 0.017 \\
\hline Feed or check diaper promptly $\dagger$ & 44 & 66.7 & 24 & $36.4^{*}$ & $<0.001$ & 52 & 80.0 & 35 & 53.8 & $<0.001$ \\
\hline Hold and soothe immediately $\dagger$ & 15 & 22.7 & 7 & $10.6^{\star \star}$ & 0.021 & 18 & 27.7 & 18 & 27.7 & 1.000 \\
\hline Check infantís bed and/or clothes & 9 & 13.6 & 16 & 24.2 & 0.118 & 9 & 13.8 & 12 & 18.5 & 0.581 \\
\hline Others & 4 & 6.1 & 19 & 28.8 & 0.001 & 7 & 10.8 & 12 & 18.5 & 0.302 \\
\hline \multicolumn{11}{|c|}{ Efforts to encourage good sleep in infant (multiple answers, $n=136$ ) } \\
\hline Wake infant longer in the daytime & 9 & 12.9 & 9 & 12.9 & 1.000 & 11 & 16.7 & 3 & 4.5 & 0.039 \\
\hline Play with infant or stimulate in the daytime & 19 & 27.1 & 34 & 48.6 & 0.003 & 23 & 34.8 & 27 & 40.9 & 0.481 \\
\hline Set regular bedtimes for daytime naps & 1 & 1.4 & 2 & 2.9 & 1.000 & 1 & 1.5 & 4 & 6.1 & 0.375 \\
\hline Settle to sleep at the same place & 28 & 40.0 & 43 & $61.4^{\star \star}$ & 0.008 & 23 & 34.8 & 26 & 39.4 & 0.508 \\
\hline Set regular bedtime and waking time & 14 & 20.0 & 28 & $40.0^{\star}$ & 0.007 & 12 & 18.2 & 16 & 24.2 & 0.424 \\
\hline Make the bedroom dark and quiet & 52 & $74.3^{\star \star}$ & 56 & 80.0 & 0.344 & 38 & 57.6 & 50 & 75.8 & 0.008 \\
\hline Feed fully and check diaper before bedtime & 54 & 77.1 & 50 & 71.4 & 0.523 & 52 & 78.8 & 49 & 74.2 & 0.607 \\
\hline $\begin{array}{l}\text { Being present at bedtime to help infant go } \\
\text { to sleep } \dagger\end{array}$ & 42 & 60.0 & 43 & 61.4 & 1.000 & 32 & 48.5 & 37 & 56.1 & 0.405 \\
\hline Letting infant fall asleep alone & 2 & 2.9 & 7 & 10.0 & 0.125 & 5 & 7.6 & 2 & 3.0 & 0.250 \\
\hline Setting bedtime routines & 4 & 5.7 & 4 & 5.7 & 1.000 & 5 & 7.6 & 9 & 13.6 & 0.289 \\
\hline $\begin{array}{l}\text { Placing stuffed toys and/or towel next } \\
\text { to infant at bedtime/during sleep }\end{array}$ & 2 & 2.9 & 8 & 11.4 & 0.070 & 3 & 4.5 & 5 & 7.6 & 0.625 \\
\hline Others & 3 & 4.3 & 5 & 7.1 & 0.625 & 6 & 9.1 & 4 & 6.1 & 0.727 \\
\hline None of the above & 3 & 4.3 & 1 & 1.4 & 0.500 & 3 & 4.5 & 3 & 4.5 & 1.000 \\
\hline
\end{tabular}

McNemar's test was used for pre/post comparison with each group. $\chi^{2}$ test was used for comparison between the two groups.

${ }^{*} P<0.10 ;{ }^{*} P<0.05$ vs control group.

†Undesirable behaviors for infant good sleep.

control group (69.5\%) responded at the follow-up stage of the questionnaire. There was no difference in the response rate between the two groups.

\section{Measurements}

Parental behaviors (sections 4 and 6, appendix 2), infant sleep problems such as night waking (section 5, appendix 2), and sleep parameters (section 1 and 2, appendix 2) were measured in mothers and infants. To evaluate parental behavior and infant sleep problems, the proportion of mothers who selected each item (which consisted of multiple answers) in the questionnaire was calculated and pre/post comparison was conducted in the intervention group and in the control group, respectively. Comparisons were then made between the intervention group and the control group at baseline of the survey and at the follow-up.

\section{Data Analysis}

For analysis, Student's $t$ test, ANOVA, McNemar's test, and the $\chi^{2}$ test were conducted using the Statistical Package for the Social Sciences (SPSS version 12.0J for Windows). The level for statistical significance was set at $P<0.05$, while a tendency toward a difference was considered at $P<0.1$.

\section{Results}

Table 1 shows the baseline characteristics of the infants and mothers, which were assessed at the first stage of the study. No significant differences were observed between the intervention group and the control group at baseline. Table 2 shows parental behavioral changes in the intervention group and the control group assessed at the 3-month follow-up stage. The mothers in the intervention group showed significant behavioral improvement relative to the control group. The incidence of undesirable parental behaviors likely to reinforce infant night waking patterns decreased. Thus, "feed or check diaper promptly" behavior decreased from $66.7 \%$ at the preintervention stage to $36.4 \%$ post-intervention $(P=0.001)$, while "hold and soothe immediately" parental behavior decreased from $22.7 \%$ to $10.6 \%(P=0.021)$. In addition, efforts to shape favorable sleep patterns in infants were found to have increased in the intervention group. Desirable behaviors such as "play with infant or stimulate in the daytime" $(P=0.003)$, "settle to sleep at the same place" $(P=0.008)$, and "set regular bedtime and waking time" $(P=0.007)$ were significantly increased.

Desirable parental behavioral changes also were observed in the control group. "Feed or check diaper promptly" behavior 
decreased from $80.0 \%$ to $53.8 \%(P=0.001)$, and "wait for a while without responding" behavior increased from $30.8 \%$ to $49.2 \%(P=0.017)$. However, the magnitude of these changes was less than those in the intervention group. Comparisons between the experimental groups showed that "feed or check diaper promptly" behavior as well as "hold and soothe immediately" behavior had a tendency toward reduction in the intervention group relative to the control group at the follow-up stage (although both these behaviors did not significantly differ to the pre-intervention stage). In addition, an unfavorable and significant decrease in the "wake infants longer in the daytime" parameter was detected in the control group $(P=0.039)$.

Table 3 shows the changes in the incidence of infant sleep problems at the follow-up stage of the study relative to the initial assessment phase. The proportion of the "night waking infants" (infants who woke more than once between midnight and 6:00 AM) significantly increased from 53.0\% to $66.7 \%$ in the control group $(P=0.022)$, while no difference was found in this parameter for the intervention group. "Frequent night waking and crying at night" showed a tendency toward increase (from $8.1 \%$ to $19.4 \%$ ) in the control group $(P=$ $0.065)$, while no difference was detected in the intervention group. The proportion of infants who showed "difficulty settling" decreased in both groups.

With regard to the sleep patterns of infants and mothers, a number of changes were detected in both groups from baseline to follow-up. Overall, the mean bedtime of the infants was found to be 40 minutes earlier (moving back from 10:23 PM to 9:43 PM), while the number of night wakings increased from a mean of 0.97 to $1.24(P<0.05)$. The mean number of naps decreased by 0.25 from 2.49 to 2.24 , and the mean total nap time was reduced by 68 minutes from 235.88 minutes to 167.71 minutes. The bedtime of mothers was found to be 17 minutes earlier (moving back from 12:03 AM to 11:46 PM), and the rising time also was earlier by 28 minutes (moving back from 7:33 AM to 7:05 AM). These changes did not significantly differ between the intervention and control groups.

\section{Discussion}

The findings in this study show that a simple intervention strategy that involved providing mothers with an educational booklet was effective in improving parental ability to promote healthy sleep patterns in their infants. By using this approach, we found that parental behaviors were significantly modified. Undesirable parental behaviors that reinforce night waking in infants were significantly reduced, while desirable behaviors beneficial for infant sleep were increased. The magnitudes of the behavioral changes were greater in the intervention group relative to the control group (note, however, that improvements were observed also in the control group). In a previous study that involved the same subjects, it was found that various undesirable parental behaviors had a negative effect on sleep problems in infants. ${ }^{22}$ Theoretically, modification of such behaviors may improve sleep problems in infants. Although our results do not show an improvement in infant sleep problems following the intervention strategy, on the basis of this study, it is suggested that this may represent an effective method for limiting further deterioration in night waking. Thus, our results show that the number of "night waking infants" significantly increased in the control group, while the incidence of "frequent night waking and crying at night" showed a tendency toward increase in the control group but not in the intervention group. Our findings support a scenario whereby educational intervention, while not significantly

Table 3. Sleep-related problems in infants.

\begin{tabular}{|c|c|c|c|c|c|c|c|c|c|c|}
\hline & \multicolumn{5}{|c|}{ Intervention group $(\mathrm{IG})(\mathrm{n}=70)$} & \multicolumn{5}{|c|}{ Control group $(C G)(n=66)$} \\
\hline & \multicolumn{2}{|c|}{ Baseline } & \multicolumn{2}{|c|}{ Follow-up } & \multirow[b]{2}{*}{$P$-value } & \multicolumn{2}{|c|}{ Baseline } & \multicolumn{2}{|c|}{ Follow-up } & \multirow[b]{2}{*}{ P-value } \\
\hline & $\mathbf{N}$ & $\%$ & $\mathbf{N}$ & $\%$ & & $\mathbf{N}$ & $\%$ & $\mathbf{N}$ & $\%$ & \\
\hline Night waking ( $=135$; IG $=69, C G=66$ ) & 43 & 62.3 & 47 & 68.1 & 0.481 & 35 & 53.0 & 44 & 66.7 & 0.022 \\
\hline \multicolumn{11}{|c|}{ Sleep-related problems (multiple answers, $n=122 ; \mathrm{IG}=60, \mathrm{CG}=62$ ) } \\
\hline Difficulty settling & 18 & 30.0 & 7 & 11.7 & 0.007 & 16 & 25.8 & 9 & 14.5 & 0.039 \\
\hline Frequent night waking and crying at night & 3 & 5.0 & 7 & 11.7 & 0.219 & 5 & 8.1 & 12 & 19.4 & 0.065 \\
\hline Light (restless) sleep & 3 & 5.0 & 5 & 8.3 & 0.727 & 3 & 4.8 & 7 & 11.3 & 0.219 \\
\hline Difficulty falling asleep alone & 22 & 36.7 & 25 & 41.7 & 0.648 & 27 & 43.5 & 27 & 43.5 & 1.000 \\
\hline Sleep-wake cycle reversal & 4 & 6.7 & 1 & 1.7 & 0.375 & 3 & 4.8 & 0 & 0.0 & \\
\hline Difficulty settling after night waking & 3 & 5.0 & 4 & 6.7 & 1.000 & 1 & 1.6 & 1 & 1.6 & 1.000 \\
\hline Snoring & 8 & 13.3 & 5 & 8.3 & 0.549 & 6 & 9.7 & 8 & 12.9 & 0.727 \\
\hline Not taking daytime naps & 6 & 10.0 & 4 & 6.7 & 0.687 & 9 & 14.5 & 5 & 8.1 & 0.344 \\
\hline Others & 7 & 11.7 & 5 & 8.3 & 0.754 & 2 & 3.2 & 5 & 8.1 & 0.453 \\
\hline Nothing & 20 & 33.3 & 20 & 33.3 & 1.000 & 25 & 40.3 & 22 & 35.5 & 0.629 \\
\hline
\end{tabular}

* McNemar's test was used for pre-post comparison within each group.

Night waking was significantly increased and frequent night waking / crying tended to increase in the control group only.

Difficulty settling improved in both groups. 
reducing the incidence of existing infant sleep problems, may be beneficial as a preventative strategy to limit the progression of such problems.

The reasons underlying the transition toward desirable changes in parental behavior in the control group as this study progressed are unclear. It is possible that the multiple choice questions in the initial questionnaire highlighted possible parental behavioral options available for controlling night waking in infants, which mothers in both groups may subsequently have adopted.

The changes found in the various sleep parameters of the infants in both groups are thought to be due to normal developmental changes. Earlier bedtimes and rising times for the mother, as well as being influenced by seasonal changes from winter to spring, are likely to be positively influenced by earlier infant bedtimes.

This study has three major advantages over other studies that have been performed in this research area. First, the educational intervention method tested here is straightforward (participants in the intervention group received information that was delivered in a 10-minute presentation and a self-help booklet only) and is therefore likely to be readily accepted by health practitioners and mothers. Indeed, this intervention strategy was performed easily in the settings of the usual health check-up system. Second, subjects were local residents who were unlikely to show selection bias. The residents who participated in the study were receiving a health check-up that is legally required for 4-month-old infants. There was no difference in the response rate to the follow-up survey between the experimental groups; both were $70 \%$. Third, the intervention method involved a population-based approach. Thus, the mothers who participated did not necessarily feel they were experiencing difficulties with the sleep patterns of their infant, and therefore in general held no special interest in this issue. In view of our approach, this study in particular stands out from previous research in this area. For example, in contrast to the recruitment method employed here, the subjects in a study of primary care intervention undertaken by Adair and colleagues ${ }^{27}$ were recruited by clinic visitors. Although a randomized controlled study performed by Scott and Richards ${ }^{3}$ provided no evidence that advice and support are effective for improving infant sleep problems, it is important to note that their study subjects were recruited and selected by screening methods. Although the present study was not a strict randomized control trial, conditions of participation within the health check-up system, as well as the demographic variables, were common between groups. Comparisons in this study are therefore most likely viable between the intervention and control groups.

Despite its advantages, this study has several limitations that should be considered when interpreting its findings. First, all information regarding the independent and dependent variables were provided by self-reports from mothers, without objective verification methods or the use of a sleep diary. Second, the data lack "infant rising time" and "total sleep time" parameters. Moreover, in order to maximize research feasibility, operational definitions were not included on the questionnaire (this was deliberate so that most mothers would easily be able to answer the questions). In practice, it is difficult to design surveys that are relatively straightforward, which at the same time provide detailed accurate assessments. Third, this study used cluster-selected controls and was not a randomized trial. Further research involving a randomized control trial with more precise sleep assessment criteria ${ }^{28-30}$ is needed to confirm the effectiveness of this intervention.

\section{Acknowledgments}

This research was presented in preliminary form at the $31 \mathrm{st}$ Annual Meeting of the Japanese Society of Sleep Research.

\section{References}

1. Mindell JA. Empirically supported treatments in pediatric psychology: bedtime refusal and night wakings in young children. J Pediatr Psychol 1999;24:465-481.

2. Mindell JA, Kuhn B, Lewin DS, Meltzer LJ, Sadeh A; American Academy of Sleep Medicine. Behavioral treatment of bedtime problems and night wakings in infants and young children. Sleep 2006;29:1263-1276.

3. Scott G, Richards M. Night waking in infants: effects of providing advice and support for parents. J Child Psychol Psychiatry 1990;31:551-567.

4. Kerr S, Jowett S. Sleep problems in pre-school children: a review of the literature. Child Care Health Dev 1994;20:379-391.

5. Hiscock H, Wake M. Randomised controlled trial of behavioural infant sleep intervention to improve infant sleep and maternal mood. BMJ 2002;324:1062-1065.

6. Zuckerman B, Stevenson J, Bailey V. Sleep problems in early childhood: continuities, predictive factors, and behavioral correlates. Pediatrics 1987;80:664-671.

7. Morin CM, Hauri PJ, Espie CA, Spielman AJ, Buysse DJ, Bootzin RR. Nonpharmacologic treatment of chronic insomnia. An American Academy of Sleep Medicine review. Sleep 1999;22:1134-1156.

8. National Institutes of Health. Insomnia.1998. NIH publication 95-3801. Available at: http://www.nhlbi.nih.gov/health/public/ sleep/insomnia.pdf. Accessed June 30, 2008.

9. Ramchandani P, Wiggs L, Webb V, Stores G. A systematic review of treatments for settling problems and night waking in young children. BMJ 2000;320:209-213.

10. Kuhn B, Elliott AJ. Efficacy of behavioral interventions for pediatric sleep disturbance. In: Perlis ML, Lichstein KL, eds. Treating sleep disorders: principles and practice of behavioral sleep medicine. New Jersey, John Wiley \& Sons;2003: 415-451.

11. Richman N, Douglas J, Hunt H, Lansdown R, Levere R. Behavioural methods in the treatment of sleep disorders - a pilot study. J Child Psychol Psychiatry 1985;26:581-590.

12. Adams LA, Rickert VI. Reducing bedtime tantrums: comparison between positive routines and graduated extinction. Pediatrics 1989;84:756-761.

13. Eckerberg B. Treatment of sleep problems in families with small children: is written information enough? Acta Paediatr 2002;91:952-959.

14. Rickert VI, Johnson CM. Reducing nocturnal awakening and crying episodes in infants and young children: a comparison between scheduled awakenings and systematic ignoring. Pediatrics 1988;81:203-212. 
15. Seymour FW, Brock P, During M, Poole G. Reducing sleep disruptions in young children: evaluation of therapist-guided and written information approaches: a brief report. J Child Psychol Psychiatry 1989;30:913-918.

16. Kerr SM, Jowett SA, Smith LN. Preventing sleep problems in infants: a randomized controlled trial. J Adv Nurs 1996;24:938-942.

17. Wolfson A, Lacks P, Futterman A. Effects of parent training on infant sleeping patterns, parents' stress, and perceived parental competence. J Consult Clin Psychol 1992;60:41-48.

18. Pinilla T, Birch LL. Help me make it through the night: behavioral entrainment of breast-fed infantsí sleep patterns. Pediatrics 1993;91:436-444.

19. Fukumizu M, Kaga M, Kohyama J, Hayes MJ. Sleep-related nighttime crying (yonaki) in Japan: a community-based study. Pediatrics 2005;115(1 suppl):217-224.

20. Latz S, Wolf AW, Lozoff B. Cosleeping in context: sleep practices and problems in young children in Japan and the United States. Arch Pediatr Adolesc Med 1999;153:339-346.

21. Hayama J, Adachi Y, Nishino N, Ohryoji F. [Sleep of 4-monthold infants: bedtime, night waking and sleep problems.] Nippon Koshu Eisei Zasshi 2007;54:440-446.

22. Hayama J, Adachi Y, Nishino N, Ohryoji F. [Impact of parenting behavior relevant to infant's sleep on maternal sleep and health.] Nippon Koshu Eisei Zasshi 2008;55:693-700.

23. Adachi Y, Yamagami T. [Baby, sleep well at night.] Kenkokoudo Publishing; 2005.

24. Adachi Y, Kunitsuka K, Hayama J, Yamatsu K, Ueda M, Yamagami T. In: Okawa M, Eds. Suimin shougai taiou no arikata ni kansuru kenkyu, Heisei 15 nendo kenkyu houkokusho. Research Grant for Mental Health (No. 200300740A) from Japanese Ministry of Health, Labour and Welfare. 77-102, 2004.

25. Kerr S, Jowett S. How to help your baby sleep well. Glasgow, England: Department of Nursing \& Midwifery Studies University of Glasgow; 1993.

26. Schmitt BD. The prevention of sleep problems. Clin Perinatol 1985;12:453-457.

27. Adair R, Zuckerman B, Bauchner H, Philipp B, Levenson S. Reducing night waking in infancy: a primary care intervention. Pediatrics 1992;89:585-588.

28. Morgenthaler TI, Owens J, Alessi C, Boehlecke B, Brown TM, Coleman J Jr, Friedman L, Kapur VK, Lee-Chiong T, Pancer J, Swick TJ; American Academy of Sleep Medicine. Practice parameters for behavioral treatment of bedtime problems and night wakings in infants and young children. Sleep 2006;29:1277-1281.

29. Buysse DJ, Ancoli-Israel S, Edinger JD, Lichstein KL, Morin CM. Recommendations for a standard research assessment of insomnia. Sleep 2006;29:1155-1173.

30. Tikotzky L, Sadeh A. Sleep patterns and sleep disruptions in kindergarten children. J Clin Child Psychol 2001;30:581-591.

\section{Author Affiliations}

Yoshiko Adachi, MD, PhD

Institute of Behavioral Health

3-29-11 Ishizaka, Dazaifu-city

Fukuoka 818-0118, Japan

and

The Association for Preventive Medicine of Japan

1-19-10 Mohri, Kouto-ku

Tokyo 135-0001, Japan

Chifumi Sato, $M D, P h D$

Department of Analytical Health Science

Graduate School of Health Sciences
Tokyo Medical and Dental University

1-5-45 Yushima, Bunkyo-ku

Tokyo 113-8519, Japan

Noriko Nishino, $M D$

Kashiigaoka Rehabilitation Hospital

2-24-36 Shimobaru, Higashi-ku, Fukuoka-city

Fukuoka 810-0073, Japan

Fumitake Ohryoji, MD

Center for Public Health and Welfare

Chuo-ku, Fukuoka 2-5-1-6F Maiduru

Chuo-ku, Fukuoka-city 810-0073, Japan

Junko Hayama, MA

Institute of Behavioral Health

3-29-11 Ishizaka, Dazaifu-city

Fukuoka 818-0118, Japan

and

Institute of Comparative Studies of International Cultures and

Societies

Kurume University

1635 Mi-machi, Kurume-city

Fukuoka 839-8502, Japan

Toshiko Yamagami, $M D, P h D$

Sawara Hospital

2-2-50 Meinohama, Nishi-ku

Fukuoka-City

Fukuoka 819-0002, Japan

\section{Appendix I:}

\section{Table of Contents from the booklet "Baby, Sleep Well at Night (Akachan, Yoru Sikkari Nemutte)"}

1. Introduction

2. Normal sleep patterns in a baby by the age of 1 year / Development and changes in sleep

3. Principles for shaping good sleep in babies / Good sleep practice for baby

4. Ten things to do right now

5. Advice for newborns / How to help your newborn sleep well

6. Advice for 2-month-old babies / How to help your 2-month old baby sleep well

7. Advice for 4-month-old babies / How to help your 4-month old baby sleep well

8. Advice for 6-month-old babies /How to help your 6-month old baby sleep well

9. Advice for 1-year-olds / How to help your 1-year-old sleep well

10. How to cope with your baby crying at night

11. Let's master learning theories for your parenting: Five basic theories

12. Principles to increase your baby's desirable behaviors

13. Principles to decrease your baby's undesirable behaviors

14. Why physical punishment or anger is not effective parenting

15. Where to go when you need advice caring for your child 


\section{Appendix 2: Questionnaire}

Please answer each of the following questions based on the last 2 weeks of your daily life and routine.

1. What time do you usually get up? $\mathrm{am} / \mathrm{pm}$

How many hours do you actually sleep? hours

What time do you usually go to bed? $\mathrm{am} / \mathrm{pm} \_$not constant

2. What time does your infant usually go to bed?

$$
\mathrm{am} / \mathrm{pm} \text { not constant }
$$

How often does your infant take a nap during the day? times

How long does your infant sleep in the daytime? hours

3. How often does your infant wake up during the night (12:00 am- 6:00 am)? times__ not waking

How often do you feed your infant during the night $(12: 00 \mathrm{am}-6: 00 \mathrm{am})$ ? times

4. If you wake during the night because your infant cries, which of the following do you do? Choose all that apply.
a. wait for a while without responding
b. feed or check diaper promptly
c. hold and soothe immediately
d. check infant's bed and / or clothes
e. other

5. Does your baby have any of the following sleep related problems? Choose all that apply.
a. difficulty settling
b. light (restless) sleep
c. frequent night waking and crying at night
d. difficulty falling asleep alone
e. sleep-wake cycle reversal
f. difficulty settling after night waking
g. snoring
h. not taking a daytime sleep
i. other
j. nothing

6. Do you go to any efforts to encourage good sleep in your infant? Choose all that apply.

a. wake infant longer in the daytime

b. play with infant or stimulate in the daytime

c. set regular bedtimes for daytime sleep

d. settle to sleep at the same place

e. set regular bedtime and waking time

f. make the bedroom dark and quiet

g. feed fully and check the diaper before bedtime

$\mathrm{h}$. being present at bedtime to help infant go to sleep

i. letting infant fall asleep alone

j. setting bedtime routines

k. placingstuffed toys and/ortowelnext to infantatbedtime during sleep

1. other

m. nothing
7. Do you have any sleep problems excluding disruption related to the care of your infant? Choose all that apply
a. light (restless) sleep
b. difficulty waking up in the morning
c. daytime sleepiness
d. difficulty falling asleep
e. sleep deprivation
f. difficulty staying asleep
g. headache after waking
$\mathrm{h}$. waking too early in the morning
i. other
j. nothing

8. Do you have any health problems? Choose all that apply
a. stiff shoulder
b. lower back pain
c. fatigue
d. pain in arm or hand
e. headache
f. irritation
g. susceptibility to catching colds
h. anxiety
i. depressed mood
j. lack of spontaneity
k. having medical treatment
1. low appetite (loss of appetite)
m. other
n. nothing

9. Do you have any problems relating to the care of your infant, excluding sleep? Mark all that apply
a. feeding
b. urination and defecation
c. regular schedule
d. growth
e. development
f. relation to sibling
g. other
h. nothing

10. How do you feel about caring for your infant now? Choose up to 3 answers.
a. joyful
b. tired
c. it has benefited me
d. rewarding
e. favorable (desirable)
f. annoying
g. uncomfortable
h. lacking in self-confidence
i. burdened
j. it is easier than I expected 